\title{
Tratamiento endoscópico de la displasia de bajo grado
}

\author{
Rafael Barreto-Zúñiga* \\ Departamento de Endoscopia, Instituto Nacional de Ciencias Médicas y Nutrición Salvador Zubirán, Ciudad de México, México
}

Diagnóstico temprano y tratamiento oportuno de lesiones premalignas han demostrado disminuir la mortalidad en pacientes con enfermedad inflamatoria intestinal (EII), esófago de Barrett (EB) y metaplasia intestinal gástrica (MIG). Endoscopia y patología de biopsias aleatorias y/o dirigidas permanece siendo el estándar de oro para diagnóstico y tratamiento oportuno de displasia asociada en estas enfermedades. Además, hay continuas actualizaciones en la literatura y congresos, relacionadas con el diagnóstico, vigilancia y tratamiento. Esta breve revisión discute principalmente las recomendaciones en el tratamiento de displasia de bajo grado (DBG) asociada en EB, Ell, y MIG. Terapia endoscópica tal como la ablación por radiofrecuencia (RFA) y/o resección endoscópica mucosa (REM) y disección endoscópica submucosa (DES) se utilizan de manera satisfactoria en tratamiento de displasia, adenocarcinoma intramucoso (AEIM) relacionada a EB y MIG. En contraste, la intervención endoscópica en displasia asociada a Ell actualmente solo se utiliza para lesiones de tipo polipoide, mientras que lesiones planas a la fecha se tratan aun mediante colectomia'.

Displasia en esófago: El objetivo del tratamiento en displasia asociada en EB (DBG/DAG) es reducir inflamación mucosa, control de displasia y prevenir la progresión hacia adenocarcinoma. Terapias endoscópicas (p. ej., ablación con radiofrecuencia (RFA) o resección endoscópica mucosa (REM) generalmente son efectuadas seguidas de vigilancia endoscópica. Existen cuatro categorías fundamentales para manejar y/o tratar condiciones de interés (p. ej., estadio 1 de early esophageal cancer [EEC], EB o displasia): 1) terapias farmacológicas; 2) vigilancia endoscópica; 3) terapias endoscópicas o terapias endoscópicas asistidas, y 4) cirugía. Estas estrategias se podrían sobreponer con algunas a de las condiciones de interés. Por ejemplo, la terapia con inhibidor de bomba de protones no es el tratamiento de EEC, pero podría reducir el riesgo de displasia y EEC entre sujetos con EB. Estas terapias podrían utilizarse en combinación (p. ej., terapia farmacológica y vigilancia para EB) dependiendo de la progresión de la enfermedad ${ }^{2}$. DBG permanece siendo un reto en el manejo clínico. Algunos estudios, sugieren un incremento en la progresión de DBG a alto grado y/o CIM (cáncer intramucoso). En especial cuando se confirma por dos patólogos expertos en EB y que el diagnóstico persista por más de 1 endoscopia. La evidencia soporta que la ablación endoscópica como tratamiento de elección en estos pacientes, aunque vigilancia permanece como una opción viable ${ }^{3}$. La terapia endoscópica es la modalidad de elección en el tratamiento de DBG y alto grado (DAG) y de CIM. Sin embargo, poco se sabe acerca de la eficacia del tratamiento a largo plazo para la remisión completa de metaplasia intestinal (CRIM). El objetivo principal de este estudio de cohorte de una base de datos de EB asociado fue el identificar falla en los predictores de CRIM en pacientes con EB asociado a DAG/ y CIM entre 2008-2018 en un centro de tercer nivel canadiense. El otro objetivo fue comparar eventos adversos con la terapia endoscópica. Un total de 328 pacientes con EB, el $28 \%$ fueron diagnosticados con DAG y $72 \%$ CIM, 
el tratamiento consistió con ablación con RFC, resección endoscópica mucosa (REM) o avulsión con calor. El total de CRIM fue del 63\%; 67\% DAG y $61 \%$ para CIM. El tiempo promedio para lograr CRIM fue 20 meses (rango de 1-82 ). En conclusión, pacientes mayores, aquellos con hernia hiatal, y segmento largo de EB son predictores de la falla de CRIM con terapia endoscópica. Estos pacientes podrían necesitar terapias más agresivas en orden de lograra $\mathrm{CRIM}^{4}$. Focal RFA en islotes de EB utilizando un régimen simplificado $\left(3 X 15 \mathrm{~J} / \mathrm{cm}^{2}\right.$, si limpiar). En este estudio se comparó el régimen de protocolo simplificado de $12 \mathrm{~J} / \mathrm{cm}^{2}$ vs. standard en segmento corto de EB (2015-2019). De 139 pacientes 10 se sometieron a protocolo estándar. Al comparar el método simplificado vs. standard se concluye: es un tratamiento efectivo para tratamiento de segmento corto del EB ${ }^{5}$. En este estudio retrospectivo ingles de 2008-2019 se evalúa la seguridad y efectividad de RFC. Se evalúa la remisión completa de CRIM remisión de displasia (CRD), recurrencia del EB, DAG y CIM. Las técnicas de RFC fueron circunferencial y focal. Como resultado de estos 239 incluidos con una edad media de 66.6 años, predominio masculino con un Praga promedio de C3M6, un 58.2\% tuvieron REM previo a RFC. En conclusión, este estudio muestra que la RFA es efectiva en ofrecer erradicación de EB y displasia con un razonable perfil de seguridad ${ }^{6}$. Resultados variables han sido reportados sobre la ablación de EB con Coagulación con Argón Plasma (APC) y aun no ha sido estandarizado. El objetivo de este estudio aleatorizado, controlado fue el evaluar el impacto de APC y post ablación con IBP para EB con displasia de bajo grado. 71 pacientes fueron aleatorizados para APC con $90 \mathrm{~W}$ o $60 \mathrm{~W}$ seguidos de $120 \mathrm{mg}$ - $40 \mathrm{mg}$ de omeprazol. Los resultados de completa ablación en el $90 \mathrm{~W} / 120 \mathrm{mg}, 90 \mathrm{~W} / 40 \mathrm{mg}$ y $60 \mathrm{~W} / 120$ mg fue confirmado en 18/23 (78\%), 15/25 (60\%), 17/23 (74\%) de pacientes a las 6 semanas y en $16 / 23(70 \%)$, $13 / 25(52 \%)$ y 15/23 (65\%) pacientes a los 2 años de tratamiento (no hubo diferencia significativa. No hubo desarrollo hacia displasia de alto grado o cáncer. En conclusión, el APC (cantidad de voltaje) y PPI dosis no impacta la eficacia y seguridad del protocolo de tratamiento en ablación de EB con displasia de bajo, fue durable en más del $90 \%$ de pacientes sin evidencia de progresión a neoplasia a largo plazo ${ }^{7}$.

Displasia gástrica: Es una lesión neoplásica y precursora de cáncer gástrico. Actualmente, la historia natural es algo incierta. Los pacientes con DBG tienen bajo riesgo de progresión a cáncer, y debido a que existen una marcada discrepancia histológica entre biopsias y especímenes endoscópicos, la REM se recomienda, en particular en la presencia de otros factores de riesgo (gran tamaño; tipo morfológico deprimido; eritema en su superficie; desnivel marcado de la mucosa, ulcera o erosión; histología tubular o túbulo-vellosa). No hay duda de que la DAG requiere REM (Fig. 1). En contraste para la DBG existen pocas guías de manejo, debido a su bajo riesgo a CG, algunos recomiendan vigilancia anual con biopsia, mientras que otros recomiendan REM debido a que el diagnóstico histológico basado en biopsia podría ser un error de muestra ${ }^{8}$. En este estudio con roedores se comprueba la incidencia de displasia gástrica fue mayor entre los roedores infectados con HP y deficiencia de hierro (50\%), comparado con aquellos infectados que se mantenían con dietas adecuadas de hierro $^{9}$. En un estudio de cohorte chino (Beijing) de 1980-2018 con un total de 4,056 sujetos en seguimiento con una media de 42 meses con diagnóstico inicial de DBG (LGD). Un 40\% tuvieron más de 3 endoscopias. Durante este periodo, $44 \%$ y $53.2 \%$ pacientes con lesiones presentaron regresión 0 persistencia respectivamente, únicamente 116 pacientes progresaron a enfermedad más avanzada (50 para DAG [HGD] y 60, GC respectivamente) su tiempo promedio de progresión fue de 3.89 años. Con estos datos, se considera que si se focaliza alguna lesión habrá que hacer resección endoscópica ${ }^{10}$. Estudios han demostrado que gastritis atrófica e infección por HP pueden ser altamente predecibles con alto grado de precisión basado sobre patrones de la micro-vasculatura. En este estudio coreano presentado en la sección de poster. La gastritis atrófica es una lesión precursora de cáncer gástrico y la infección por $\mathrm{H}$. pylori es un factor de riesgo bien conocido para cáncer gástrico. Una infección de HP presenta cambios en la mucosa gástrica en la vénulas colectoras, capilares y glándulas circundantes. El objetivo de este estudio fue validar la utilidad de imágenes endoscópicas para predecir gastritis atrófica y estatus de infección por HP para evitar biopsias innecesarias. Estudio prospectivo en 2 centros académicos coreanos. 45 pacientes asintomáticos entre 20 y 80 años se les efectuó endoscopia, los hallazgos se clasificaron dentro de tres tipos de acuerdo con la disposición o arreglo regular de los patrones en vasos colectores (vénulas, pequeños vasos colectores gástricos) (RAC) y red subepitelial capilar (SECN) ${ }^{11}$.

Displasia en colon: El riesgo a largo plazo de DAG y cancer colorectal (CCR) despues de DBG en Ell se desconoce. En un estudio cohorte, retrospectivo 


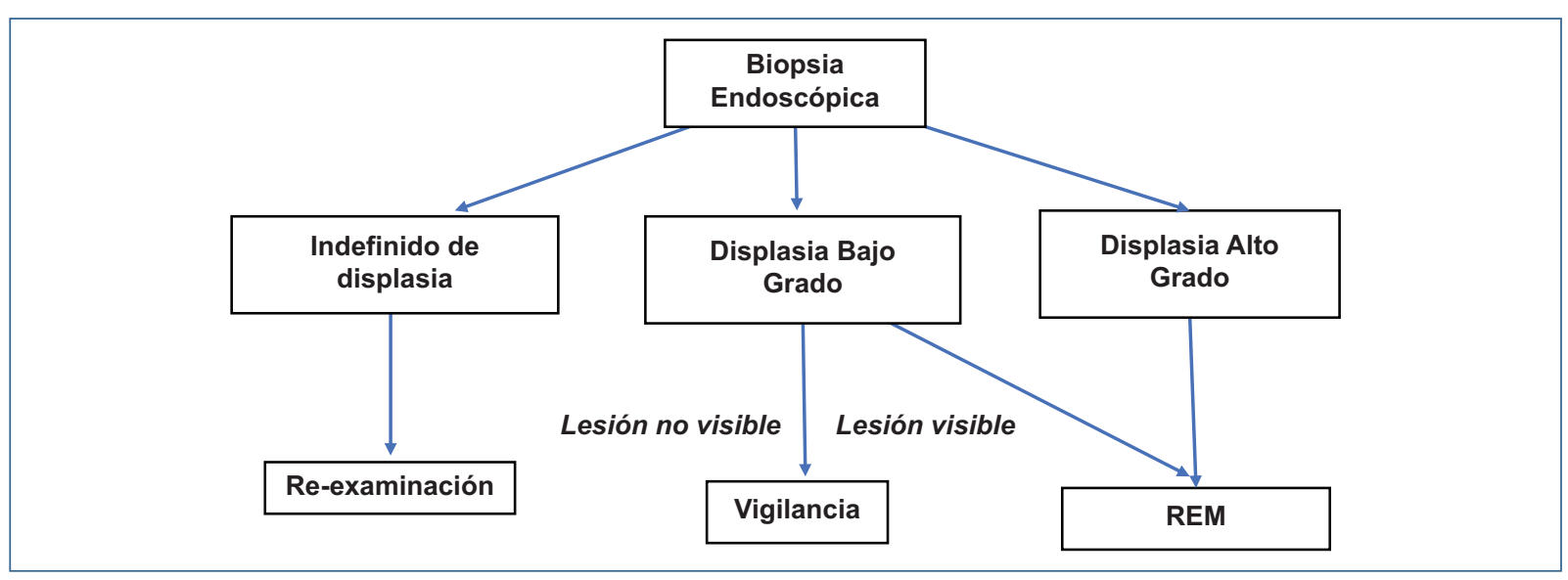

Figura 1. Estrategia de Tratamiento propuesto para displasia diagnosticada mediante biopsia endoscópica. Erradicación de Helicobacter pylori se recomienda después de REM.

holandes de 1991-2016, se identificaron 4284 pacientes con DBG. La incidencia acumulada a neoplasia avanzada fue de $21.7 \%$ despues de 15 años. Edad avanzada de DBG (> 55 años) sexo masculino y vigilancia en un centro de tercer nivel fueron factores independientes de riesgo después de $\mathrm{DBG}^{12}$. Polipectomía con asa fría utilizada por asa convencional con alambre en una miniasa parecería ser útil clínicamente parecería ser útil en pólipos colorectales pequeños. El objetivo de este estudio coreano. la polipectomia con asa fría (CSP por siglas en ingles de Cold Snare Polypectomy) se ha establecido como una técnica para la resección de pólipos en colon y recto por arriba de $10 \mathrm{~mm}$ de tamaño sin utilizar unidad elctro-quirurgica. Sin embargo, existe una falta de estudios comparativos utilizando asa delgada (microfilamento) designada para CSP y asa gruesa mini-asa durante CSP. Este es un estudio prospectivo, aleatorizado y controlado entre julio y octubre del 2017 un total de 113 pólipos neoplásicos de cáncer de colon y recto (5-8 $\mathrm{mm}$ de diámetro) fueron removidos por CSP e incluidos en el estudio. Los principales puntos para evaluarse son el porcentaje de recesión completa. Un total de 113 pólipos elegibles para CSP (asa monofilamento) grupo de 48 sujetos y asa gruesa de 47 . No existieron diferencias significativas en ambos grupos. En conclusión polipectomia con asa fría con asa monofilamento i asa gruesa es útil para pólipos pequeños ${ }^{13}$. Este estudio retrospectivo, evaluó resultados de REM en un centro brasileño de 1038 sujetos de 20162018: neoplasia intraepielial de bajo grado en 592 (57\%), Neoplasia intraepitelial alto grado en 201 (19.3\%), bajo grado itraepietlial en pólipos aserrados:
$51(4.9 \%)$ con $44.2 \%$ de lesiones protruidas $n=459$ y $559(55.8 \%)$ tipo superficial ${ }^{14}$. Estudio retrospectivo de lesiones deprimidas con estudio de 485 lesiones (478 pacientes), con displasia de bajo grado (neoplasia intraepitelial de bajo grado) en 292 casos (60.2\%) en lesiones por arriba de $5 \mathrm{~mm}$ (61.8\%). Los pólipos aserrados son considerados para el Desarrollo de displasia por la vía de neoplasia aserrada, estos pólipos son difíciles de detectar y con frecuencia su resección es incompleta en este estudio retrospectivo coreano se evalúa diagnóstico y tratamiento en 2015-2018 con pólipos aserrados mayores de $10 \mathrm{~mm}$ con resección endoscópica de un total de 59 Pólipos aserrados (51 pacientes) la media de tamaño $15.0 \mathrm{~mm}$ y cerca del $80 \%$ en colon derecho, treinta de 59 pólipos (50.9\%) presentaron displasia por histopatología (21 de bajo grado, 8 DAG, y 1 carcinoma in situ). En conclusión, displasia en lesiones aserradas frecuentemente encontrada en pacientes viejos y con pólipos de gran tamaño. Por lo tanto, todos los pólipos serrados deberán ser resecados completamente para prevención de recurrencia local y cáncer de intervalo ${ }^{15}$.

\section{Financiamiento}

Los autores no recibieron patrocinio para llevar a cabo este artículo.

\section{Conflicto de intereses}

Los autores declaran no tener conflicto de intereses alguno. 


\section{Bibliografía}

1. Karamchandani DM, Zhang Q, Liao XY, et al. Review; Inflammatory bowel disease-and Barrett's esophagus-associated neoplasia: the old, the new. And the persistent struggles. Gastroenterology Report 2019;7(6):379-395

2. Hamel C, Ahmadzai N, Beck A, et al. Screening for esophageal adenocarcinoma and precancerous conditions (dysplasia and Barrett's esophagus) in patients with chronic gastroesophageal reflux disease with or without other risk factors: two systematic reviews and one overview of reviews to inform a guideline of the Canadian Task Force on Preventive Health Care (CTFPHC). BMC Hamel et al. Systematic Reviews (2020) 9:20 https://doi.org/10.1186/s13643-020-1275-2

3. Falk GW, Current Management of Low-Grade Dysplasia in Barrett Esophagus. Gastroenterol and Hepatol 2017;(3)4:221-225

4. Kobayashi R, Calo N, Li S, et al. Factors Associated with lack of complete Remission of Intestinal Metaplasia in Patients with Barrett's Esophagus Associated High Grade Dysplasia and Intramucosal Cancer. In DDW 2020 Chicago Illinois. USA; Mo1293.

5. Diaz LI, Kukreja K, Mony S, et al. Simplified Versus Standard Regimen for Focal Radiofrequency Ablation of Short Segment Dysplastic Barrett's Esophagus are Equally Effective: Should we Keep It Simple? In ASGE. DDW 2020 Chicago Illinois USA. MO 1291.

6. White J, Ortiz-Fernandez S, Santiago-Garcia J, et al. RFA for Dysplastic Barrett's Esophagus: 10-Years of Experience from The East Midlands. Endo2020, Rio de Janeiro Brazil P362.

7. Wronska E, Polkowski M, Orlowska J, et al. Argon Plasma Coagulation for Barrett's Esophagus with Low Grade dysplasia: Randomized Trial with Long-Term Follow-Up Evaluating the Impact of Power Setting and Proton Pump Inhibitor Dose. In ASGE. DDW 2020 Chicago Illinois USA. MO-68.
8. Sung JE. Diagnosis and management of gastric dysplasia. Review. Korean J Intern Med 2016;31: 201-209.

9. Noto MJ, Carmichael J, Piazuelo MB, et al. Iron Deficiency Promotes Helicobacter Pylori-Induced Carcinogenesis Via Targeted Disruption of Inflammatory and Metabolic Pathways. 18-2020 AGA. DDW Chicago IIlinois USA.

10. Xiao S, Lu H, Yin Z et al. Risk Factors for Developing High-Grade Dysplasia or Gastric Cancer In patients with gastric Low-Grade Dysplasia: Results From a Large Surveillance Cohort from 1980 to 2018. OP008. ENDO 2020, Rio Janeiro Brazil Department of Gastroenterology Beijing, China.

11. Ko W, Woon $\mathrm{Y}, \mathrm{Kim} \mathrm{H}$, et al. Accuracy of diagnosis of chronic gastritis and HP infection Through Microvascular Pattern. ENDO 2020 (poster) P005 Rio de Janeiro Brazil.

12. de Jong M, van Tilburg $\mathrm{S}$, Nissen $\mathrm{H}$, et al. Long-term Risk of Advanced Neoplasia After Colonic low-grade Dysplasia in Patients With inflammatory Bowel Disease: A Nationwide Cohort Study. Journal of Crohn's and Colitis, 2019, 1485-1491. Doi.10.1093/ecco-jcc/jjz114.

13. Chung K, Jung Y, Sin Cho Y, et al. Comparison of the Clinical Efficacy of the Cold Snare polypectomy Using Thin Wire-mini-Snare and Thick Wire mini-Snare for Small Size $(5-8 \mathrm{~mm})$ colorectal polyps. P048. Endo 2020 Rio de Janeiro Brazil.

14. Rio de Janeiro P079 Endoscopic Mucosal Resection of a Flat and sessile colorectal lesions: Analysis of Endoscopic and Histopathological Aspects of 1038 cases in the last 2 years. Parada A, Degiovani M, Varca N, et al. ENDO 2020, Rio de Janeiro Brazil P079.

15. P230: Kim W, Park S, Kim S, et al. Clinical and Endoscopic Findings Associated with Dysplasia in Sessile Serrated Adenomatous/Polyp. ENDO 2020. 